\title{
Testing of the "Tor Vergata" Fabry-Pérot interferometer prototype
}

\author{
Luca Giovannelli, Francesco Berrilli, Martina Cocciolo, Dario Del Moro, Alberto Egidi, \\ Roberto Piazzesi and Marco Stangalini \\ Dipartimento di Fisica, Università di Roma "Tor Vergata", Via della Ricerca Scientifica, \\ I-00133 Rome, Italy
}

\begin{abstract}
In this contribution we present preliminary mechanical and optical tests of the Fabry-Pérot interferometer prototype developed at the "Tor Vergata" University Solar Physics Laboratory. Fabry-Pérot narrow filters are of great interest for the study of extended astronomical sources, such as the solar photosphere and chromosphere. The high transparency of the instrument allows for the necessary high time-resolution for fast dynamic processes observations. A dedicated software has been developed to control both coarse and fine piezo-actuated movements, allowing for fast (1ms) tuning capabilities. General mechanical behaviour has been tested for use at the focal plane of ground based telescopes and in the perspective of a new space-qualified prototype.
\end{abstract}

Keywords: Fabry-Pérot Interferometer, space telescopes, spectroscopy, solar physics

\section{INTRODUCTION}

A Fabry-Pérot (FP) is an instrument based on multiple beam interferometry. It consists of two high reflectivity flat plates which create an optical cavity in which an incoming beam undergoes multiple reflections. The constructive interference and the consequent wavelength selection depend on the optical path difference, i.e. on the gap spacing $d$ between the plates.

In this way, the interferometer allows transmission of light at well-defined wavelengths. A spectrometer based on tunable filter is a solution to the the modern spectroscopy problem of collecting both spatial and spectral information. Every image acquired is nearly monochromatic and it is possible to rapidly change the wavelength of the observation varying the gap width. Fabry-Pérot tunable filters are of exceptional interest in high spectral resolution imaging for both ground-based and space applications ${ }^{1}$ since they allow the scanning of spectral lines in an extended range of wavelengths.

The prototype here presented has been developed as part of the study for the narrow band channel of the ADAHELI mission. The ADvanced Astronomy for HELIophysics (ADAHELI) is a solar satellite designed to investigate the dynamics of solar atmosphere as part of the Italian Space Agency (ASI) program. A proposal based on ADAHELI was submitted to ESA S-class Mission 2012 Call. Although our main goal was to realize an instrument for space use, other ground-based applications have been considered, expecially in the frame of the new 4-meter class of solar telescopes (e.g. ATST, EST).

In order to understand the feasibility of a space Fabry-Pérot interferometer, a laboratory prototype has been tested for general mechanical behavior.

\section{THE ADAHELI MISSION}

ADAHELI is a small-class low-budget satellite mission with the main aim to monitor solar flares and to study the dynamics of different layers of the solar atmosphere. ${ }^{2,3}$ ADAHELI design has completed its Phase-A feasibility study in December 2008, in the framework of ASI (Agenzia Spaziale Italiana) 2007 Small Missions Program. The University of Rome "Tor Vergata" is the leading scientific institution of the project and CGS SpA is the leading industrial partner.

\footnotetext{
Further author information:

Giovannelli, L.: E-mail: giovannelli@roma2.infn.it
} 
The ISODY (Interferometer for SOlar Dynamics ${ }^{4}$ ) instrument is the main payload of the satellite. It is designed to obtain high resolution spatial, spectral, and temporal polarimetric images of the solar photosphere and chromosphere. The Focal Plane Assembly ${ }^{5}$ of the ISODY instrument comprises two visible near-infrared science optical paths or channels: the Narrow Band (NB) and the Broad Band (BB) channels. The principal optical path of the NB channel includes two FP interferometers used in axial-mode and in classic mount and a filter wheel with four interference filters used to select the orders of the FPs.

A letter of intent about an advanced version of the satellite (ADAHELI+) was submitted to ESA S-class Mission 2012 Call.

\section{PROTOTYPE PARAMETERS CHOICE}
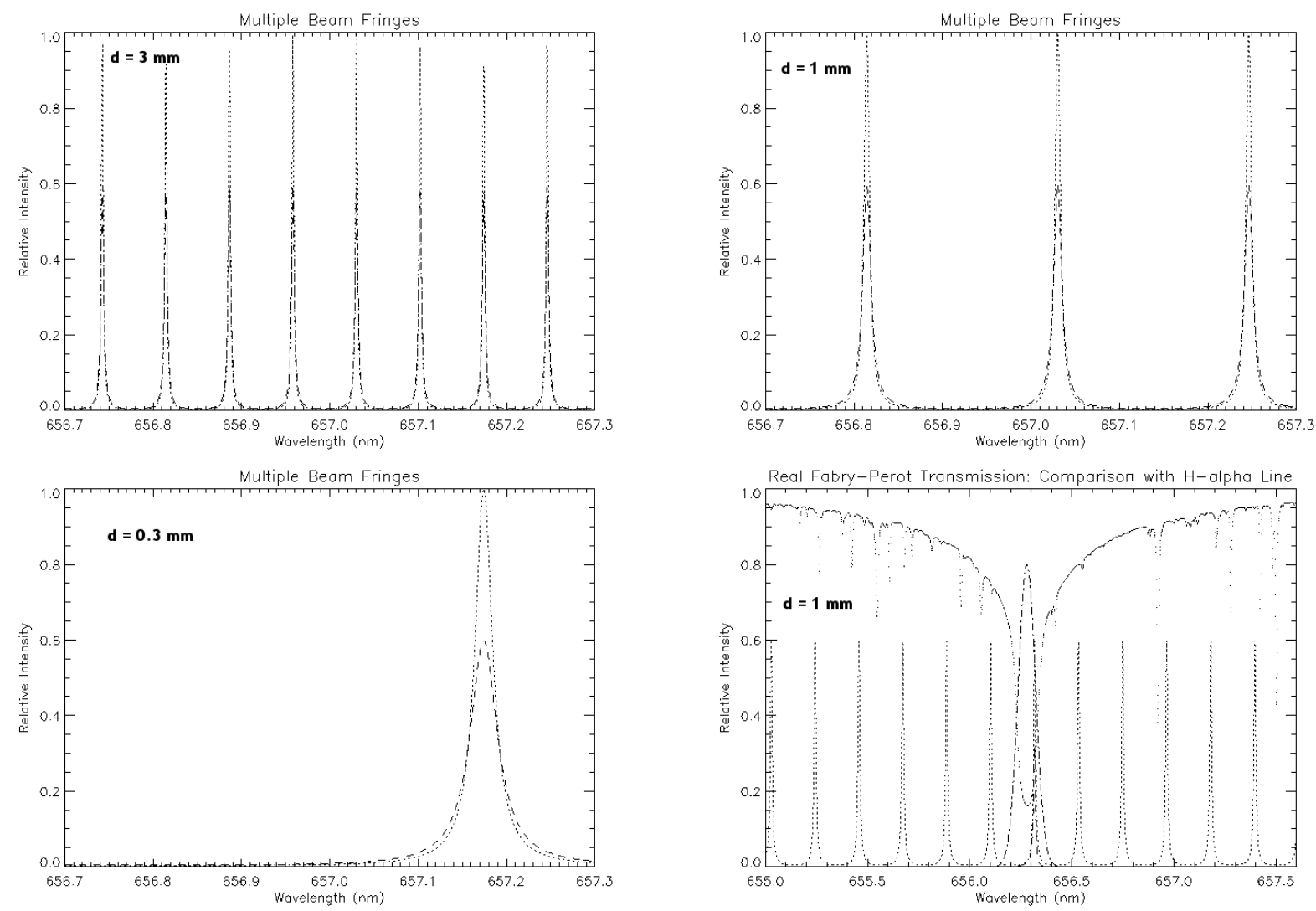

Figure 1. FP numerical simulations. Dotted: ideal FP profile; dashed: effective FP profile. The respective gap widths $d$ are indicated in the lower left corner of each panel; in the lower left panel we also plot the $H_{\alpha}$ absorption line (dotted) and the prefilter profile (dot-dashed).

\subsection{The ideal Fabry-Pérot}

If we analyze the spectral response of the instrument, we can see that transparency has several peaks, resembling a comb. The transmission profile depends on the value of the reflectivity $R$, the reflecting finesse $\mathcal{F}_{R}$ and the interference order $m^{6}$ :

$$
\begin{gathered}
\mathcal{F}_{R}=\frac{\pi \sqrt{R}}{1-R} \\
m=\frac{2 n}{\lambda} d \cos \theta
\end{gathered}
$$

The order $m$, once defined the working wavelength, depends on the refracting index $n$, the angle of incidence $\theta$ and the gap spacing $d$. The incidence angle of the beam on the instrument is usually normal, which leaves us 
with two possible ways to change $m$ : either vary the refracting index $n$ or the spacing $d$.

The reflecting finesse depends only on the reflectivity $R$ and is set choosing a suitable multilayer dielectric coating. The Free Spectral Range (FSR, is the distance between consecutive orders peaks) and FWHM of the transmission peak are then:

$$
\begin{aligned}
F S R & =\frac{\lambda}{m} \\
F W H M & =\frac{F S R}{\mathcal{F}_{R}}
\end{aligned}
$$

It is evident that the reflecting finesse $\mathcal{F}_{R}$ and the interference order $m$ are the parameters that define the characteristics of a FP-based spectrometer. The FSR is the maximum wavelength range we can scan with a single peak and sets the spectral passband of the prefilters. The FWHM defines the spectral resolution.

\subsection{The effective Fabry-Pérot}

Key issues for a plane parallel interferometer are the defects of the plates, a not perfect parallelism and the effects of a not perfectly collimated beam. They cause a broadening of the spectral profile which can be taken into account in term of finesse.

We can model the effect of a not perfectly flat plate using the spherical defect finesse $\mathcal{F}_{D S}$, where $\delta_{D S}$ is the peak to valley value of deviation from the plane surface.

Aside from systematic surface defects, a surface rugosity $\delta_{D G}$ is always present after the plate has been polished. With the hypothesis of Gaussian distributed residuals errors on the surface, it can be described with the root mean square (RMS) of the difference between the measured and the ideal surface.

The parallelism defect finesse $\mathcal{F}_{D P}$ takes into account the effect generated by not perfectly parallel plates. Here, $\delta_{D P}$ is the maximum variation of the gap spacing.

If the incident beam has a certain amount of divergence, the difference of incidence angles between inner and outer rays will also broaden the transmission profile, as a function of the solid angle of the beam $\Omega$ or the divergence angle of the beam $\theta_{D I V}$. This effect is called the aperture finesse $\mathcal{F}_{D I V}$, since the aperture of the instrument limits range of possible angles of the diverging beam. All previous definitions of finesse can be included in the effective finesse $\mathcal{F}_{E}$ which describes an effective interferometer.

$$
\mathcal{F}_{E}=\left(\frac{1}{\mathcal{F}_{R}^{2}}+\frac{1}{\mathcal{F}_{D S}^{2}}+\frac{1}{\mathcal{F}_{D G}^{2}}+\frac{1}{\mathcal{F}_{D P}^{2}}+\frac{1}{\mathcal{F}_{D I V}^{2}}\right)^{-\frac{1}{2}}
$$

This quantity has to be used instead of the reflective finesse in all previous formula for the ideal Fabry-Pérot in order to describe the effective interferometer. If we go back to equations 4 and 3, it is clear that the spectral profile is broadened by the effective finesse being smaller then the ideal reflecting finesse, thus increasing the FWHM but leaving the FSR unchanged.

The transmitted intensity for the effective case can be now computed using the effective finesse $\mathcal{F}_{E}$. The transmitted intensity equation in this way becomes:

$$
I_{T}=I_{0}\left(1-\frac{A}{1-R}\right)^{2} \frac{1}{1+\left(\frac{2 \mathcal{F}_{E}}{\pi}\right)^{2} \sin ^{2} \frac{\Delta}{2}}
$$

Broadening of the transmission profile and consequent decrease in spectral resolution is not the only effect: there is also an additional reduction in the maximum transmission intensity, so that

$$
I_{M A X} \simeq\left(1-\frac{A}{1-R}\right)^{2}\left[1-\frac{1+R}{2}\left(1-\frac{\mathcal{F}_{E}}{\mathcal{F}_{R}}\right)\right]
$$




\subsection{Numerical simulation}

We developed a software model to simulate the performance of the prototype and we used the $\mathcal{F}_{E}$ term to account for the effective behaviour of the system. We simulated three FPs, with three different gap widths $d$ : $0.3 \mathrm{~mm}, 1.0 \mathrm{~mm}$ and $3.0 \mathrm{~mm}$ (Fig.1 and Table 3.3). The broadening of the transmission profiles and the drop in transparency for the effective cases is rather evident (Peak transparency $=59.8 \%$ ).

The simulation aim was to decide which $d$ value made the instrument more suitable to scan the $H_{\alpha}$ line $\left(\lambda_{0}=\right.$ $656.3 \mathrm{~nm}$ ), taking into account the available commercial prefilter (the prefilter profile is plotted in the lower right panel of Fig.1). In a trade-off between the FSR and Resolving Power $\mathcal{R}=\lambda_{0} /$ FWHM set by the optical gap, and using equation 4 , we selected the $d=1.0 \mathrm{~mm}$ value.

\begin{tabular}{l|c|c|c|c|c|c} 
& Ideal & Effective & Ideal & Effective & Ideal & Effective \\
\hline$d[\mathrm{~mm}]$ & 0.3 & 0.3 & 1.0 & 1.0 & 3.0 & 3.0 \\
\hline $\mathcal{F}$ & 29.80 & 19.23 & 29.80 & 19.23 & 29.80 & 19.23 \\
\hline FWHM $[\mathrm{nm}]$ & 0.0241 & 0.037 & 0.0072 & 0.0112 & 0.0024 & 0.0037 \\
\hline FSR $[\mathrm{nm}]$ & 0.718 & 0.718 & 0.215 & 0.215 & 0.072 & 0.072 \\
\hline $\mathcal{R}$ & 27247 & 17576 & 90824 & 58589 & 272471 & 175767
\end{tabular}

Table 1. Parameters and results of the FP simulation runs, comparing the ideal and the effective parameters of the simulated FPs.

\section{PROTOTYPE DESIGN}

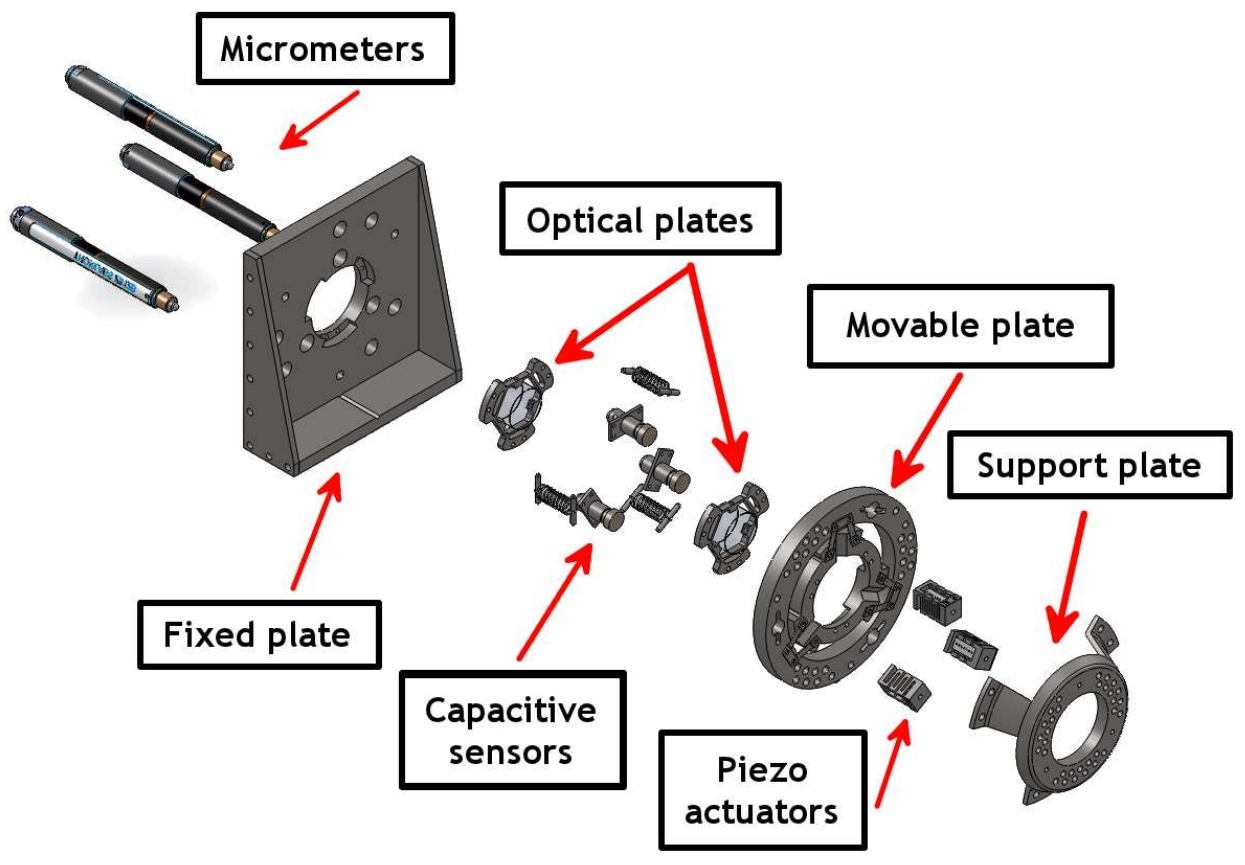

Figure 2. Prototype exploded view.

To study the feasibility of a space-qualified FP, we realized a scanning optical cavity with a gap servo control system. The main requirements for this device are to allow a fast variation of the gap within the semi-reflecting plates, while maintaining their parallelism within the tolerances. In addition, positioning repeatability must be guaranteed in order to obtain the same spectral points in successive measures.

To achieve these goals, we employ piezoelectric actuators to change the gap spacing and capacitive sensors to 
monitor its width. The combination of these two technologies in a servo controlled system grants the necessary stability and repeatability $\left(\lambda / 3000\right.$ over one day or more $\left.{ }^{7}\right)$. We have designed the optomechanics of the FP prototype to house: a pair of 1" $(25.4 \mathrm{~mm})$ partially reflecting mirrors, three micrometers, three piezoelectric actuators, three high sensitivity capacitive sensors, with a $120^{\circ}$-symmetry around the etalon (See Fig. 2). The micrometers allow for a coarse adjustment of the gap width, up to $\pm 12 \mathrm{~mm}$. The piezo-actuators allow for a finer adjustment, with a maximum extension range of $15 \mu \mathrm{m}$, while the capacitive sensors working distance is $50 \mu m$. The gap width can be varied either with the piezo-actuators or with the micrometers, since the prototype has been designed to decouple these two different adjustment by separating the movable plate into two concentric rings (see Fig. 4). The inner ring, which houses the optical surface, is controlled by the piezo-actuators, which can vary the gap size within the optomechanical specifications. The outer ring is connected to the inner ring by three V-shaped flexures, therefore a micrometer displacement moves both the inner and the outer ring, while a piezo-actuator displacement moves the inner ring only.

\subsection{Piezoelectric actuator tests}

As stated in Sec. 4, the fine adjustments of the optical plate are achieved using piezoelectric actuators. The piezo-actuators can perform sub-nanometer moves, have no rotating or sliding parts to cause friction and dissipate virtually no power in static operation. Also, they can operate in low pressure and low temperature compatible with space environments $(2 \mathrm{~K}$ and 0.5 bar). To verify the performances of the piezo-actuator, both in closed and open loop operation modes, we tested them in our laboratory. The piezo are computer controlled via a 16-bit Digital to Analog Converter (DAC), with a $0.44 \mathrm{~nm}$ minimum step. We measured the open loop behaviour feeding the DAC with a ramp from $-1 \mathrm{~V}$ to $10 \mathrm{~V}$ with $1 \mathrm{~V}$ steps (see upper left panel of Fig. 3). The maximum elongation is $14.1 \pm 0.2 \mu \mathrm{m}$, which is near the nominal value of $15 \mu \mathrm{m}$. Also, the hysteresis cycle of the piezo is evident: lower points refers to the pullout while upper points refers to the pullback. This well-known behaviour is compensated for via a closed loop control system with high-precision distance capacitive sensor. We also measured the closed loop performances (see right panels of Fig. 3) using a reduced control voltage range, but still able to cover the full range of the piezo extension. In the closed loop configuration, the maximum extension of the piezo-actuator is $14.8 \pm 0.2 \mu \mathrm{m}$, and its behaviour is strongly linear as can be derived from the linear fit and its residuals.
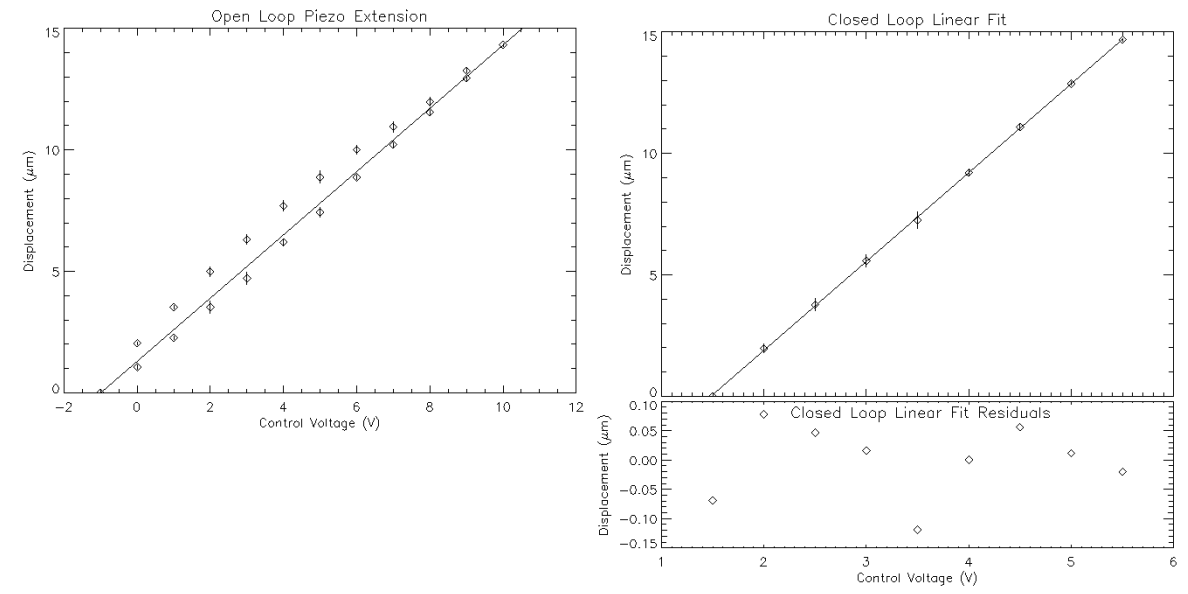

Figure 3. Piezo-actuators elongation data, from left to right: open loop hysteresis, closed loop with linear fit and related residuals.

\subsection{Initial assembly phase}

The prototype mechanical parts have been realized in-house in stainless steel. We selected stainless steel for its stiffness properties, low cost and availability. The two concentric rings of the movable plate have been realized with a maximum surface error $<40 \mu m$ over the whole surface (see Fig. 4). The mechanical parts of the 

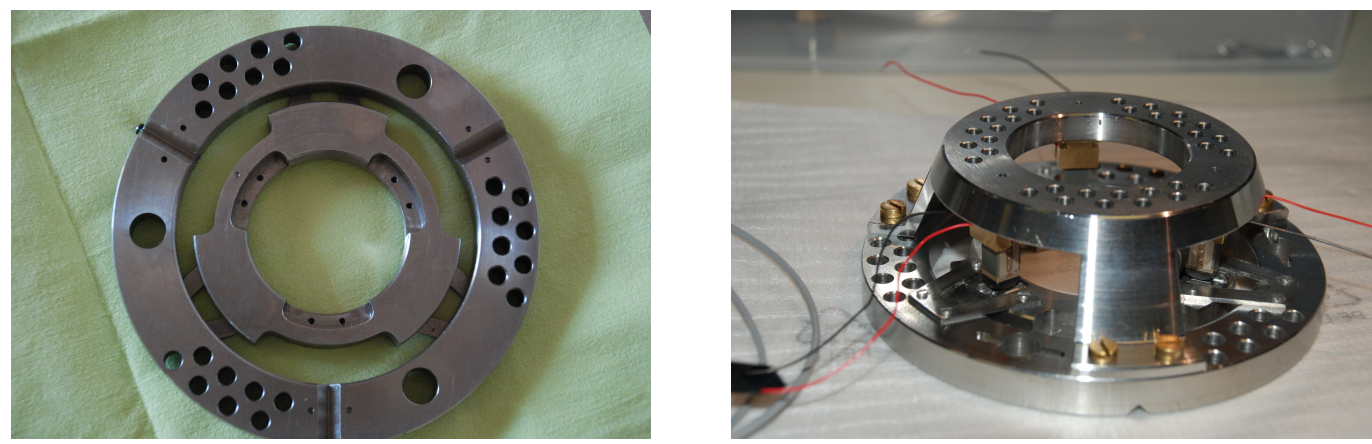

Figure 4. Movable plate: inner and outer rings (left), rings and support plate (right).

prototype has been assembled in the laboratory as well as the micrometers, the piezo-actuators, the capacitive sensors and the optical plates (Fig. 5). At present, two preliminary sets of optical plates have been manufactured by LightMachinery (www.lightmachinery.com), with $\lambda / 30$ and $\lambda / 60$ surface errors, respectively.

\section{CONCLUSIONS AND FUTURE DEVELOPMENTS}

In this work we have presented the design of a laboratory prototype Capacitance Stabilized Fabry-Pérot interferometer. First, we introduced the basic FP theory and presented the results an effective FP numerical model. From these results, we designed a FP prototype optimized for the scan of the $H_{\alpha}$ line, but also suitable for the whole visible range. We have presented the mechanical, electronic and optical characteristics of the prototype components and part of the tests we performed on them.

We have described the solution adopted in the prototype design and its assembly. In the near future, we plan to perform the full optical and spectral qualification of this prototype.

Furthermore, we are working on a space implementation of the FP in order to start space qualification tests. The design of that FP has been update to match the more demanding operating environment requirements, such as the launch stresses and the larger thermal variations.

\section{REFERENCES}

[1] Kameda, S., Murakami, G., Yoshikawa, I., Korablev, O., and Rees, D., "The Mercury Sodium Atmosphere Spectral Imager (MSASI) onboard BepiColombo/MMO," in [38th COSPAR Scientific Assembly], 38, 746 (2010).

[2] Berrilli, F., Velli, M., Roselli, L., Bigazzi, A., and the ADAHELI Team, "The adaheli (advanced astronomy for heliophysics) solar mission," Mem. SAIt 80, 251 (2009).

[3] Berrilli, F., Bigazzi, A., Roselli, L., Sabatini, P., Velli, M., Alimenti, F., Cavallini, F., Greco, V., Moretti, P., Orsini, S., Romoli, M., White, S. M., and Team, A., "The adaheli solar mission: Investigating the structure of sun's lower atmosphere," Advances in Space Research 45, 1191-1202 (2010).

[4] Greco, V., Cavallini, F., and Berrilli, F., "The telescope and the double fabry-perot interferometer for the adaheli solar space mission," in [Space Telescopes and Instrumentation 2010: Optical, Infrared, and Millimeter Wave], Oschmann, J., Clampin, M., and MacEwen, H., eds., Proc. SPIE 7731, 773142-773142-9 (2010).

[5] Berrilli, F., Cocciolo, M., Giovannelli, L., Del Moro, D., Giannattasio, F., Piazzesi, R., Stangalini, M., Egidi, A., Cavallini, F., Greco, V., and Selci, S., "The Fabry-Perot interferometer prototype for the ADAHELI solar small mission," in [Society of Photo-Optical Instrumentation Engineers (SPIE) Conference Series], Society of Photo-Optical Instrumentation Engineers (SPIE) Conference Series 8148 (Sept. 2011).

[6] Born, M. and Wolf, E., [Principles of Optics], Cambridge University Press (2002 (seventh edition)).

[7] Hicks, T. R., Reay, N. K., and Atherton, P. D., "The application of capacitance micrometry to the control of fabry-perot etalons," Journal of Physics E: Scientific Instruments 17(1), 49 (1984). 


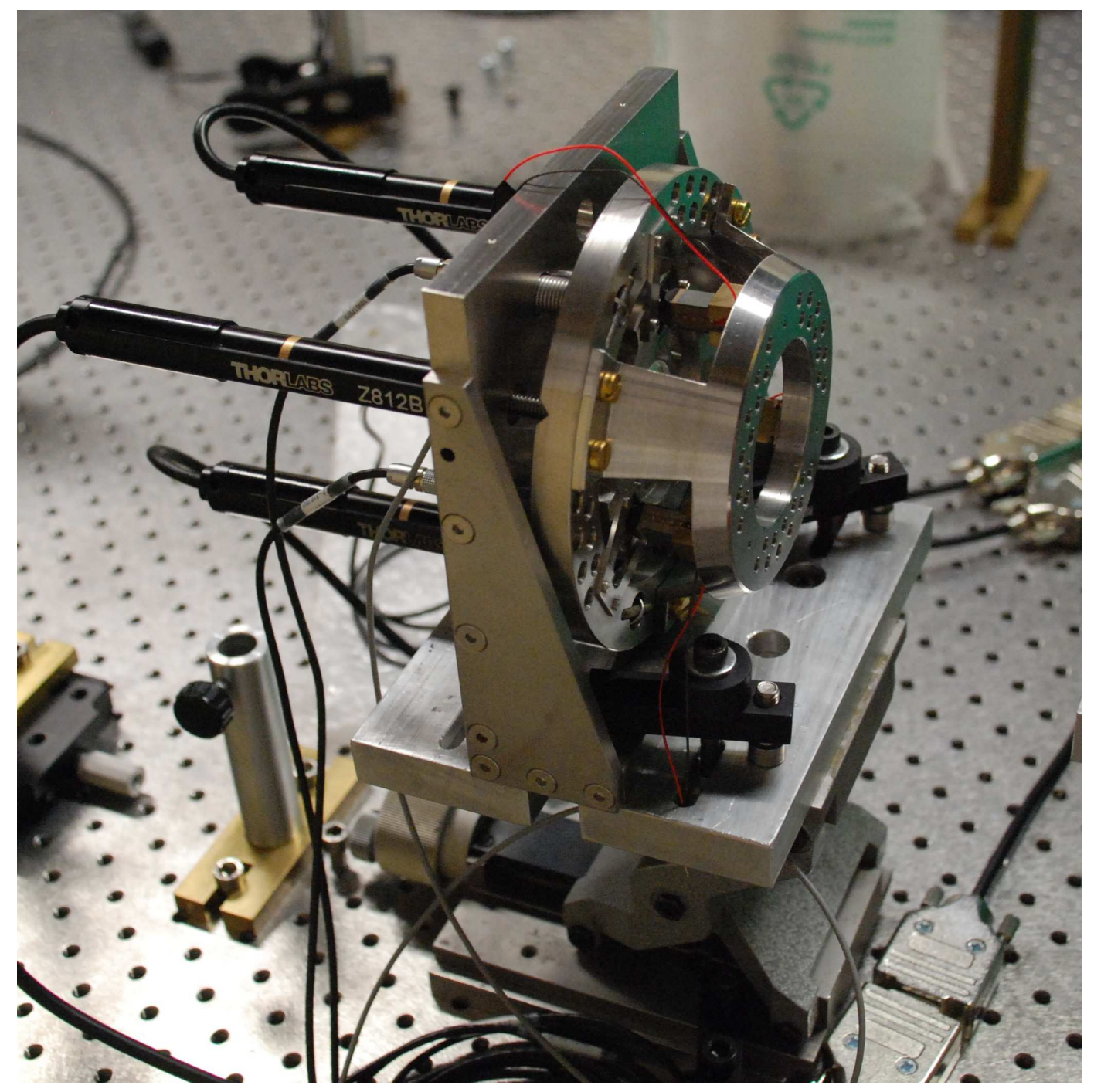

Figure 5. Assembled prototype on the optical table for mechanical and optical tests. 